\title{
DEGRADED CONFINEMENT AND TURBULENCE IN TOKAMAK EXPERIMENTS
}

\author{
G.M.D. Hogeweij \\ FOM-Institute for Plasma Physics Rijnhuizen, Association EURATOM-FOM, Trilateral Euregio Cluster, \\ P.O.Box 1207, 3430 BE Nieuwegein, The Netherlands, www.rijnh.nl
}

\begin{abstract}
After a review on the state of tokamak transport theory, the methodology to derive experimental results will be described. Examples of confinement in ohmic plasmas and the deterioration with additional healing will be given. Some examples of improved confinement modes will be discussed.
\end{abstract}

\section{SUMMARY OF THEORETICAL EXPECTATIONS}

In this section only a summary of theoretical expectations is given since in other lectures many aspects of transport theory will be treated, i.e. kinetic theory [1], classical and neoclassical transport in tokamaks [2], and the role of micro-instabilities in transport [3].

\section{I.A. Collisional Transport Theory}

Predictions of collisional transport theory in toroidal geometry, i.e. the so-called neoclassical transport (in the following denoted by subscript 'nc') can be described in short by:

a Particle diffusion: from $D^{\text {ban }}=D_{\mathrm{c}} q^{2}(R / r)^{1.5}$ at low collisionality (Banana regime) to $D^{\mathrm{PS}}=D_{\mathrm{c}}\left(1+q^{2}\right)$ at high collisionality (PfirschSchlüter) with $D_{\mathrm{c}}=\nu_{\mathrm{e}} \rho_{\mathrm{e}}{ }^{2}$. This results in typically $D^{\text {nc }} \simeq 10^{-2}$ to $10^{-1} \mathrm{~m} / \mathrm{s}$.

b Inward convection is predicted to have values $V_{\mathrm{c}}^{\mathrm{nc}}=-\epsilon^{0.5} E_{\mathrm{tor}} / B_{\mathrm{pol}}$, the so-called Ware-pinch, leading to values of typical 0.01 to $0.20 \mathrm{~m} / \mathrm{s}$. Also temperature gradients will drive an inward convection flux comparable to or smaller than the outward diffusive flux.

c Impurity fluxes are dominated by the friction with the main ion population. This implies an accumulation of the impurities at the location of highest main ion density following: $n_{\mathrm{Z}} / n_{\mathrm{Z}}(0)\left(n_{\mathrm{e}} / n_{\mathrm{e}}(0)\right)^{Z}$.

$\mathrm{d}$ The electron heat diffusivity, $\chi_{\mathrm{e}}^{\mathrm{nc}}$, should be close to the particle diffusion coefficient $D^{\text {nc }}$.

e The ion heat diffusivity, $\chi_{\mathrm{i}}^{\text {nc }}$, proportional to $\nu_{\mathrm{i}} \rho_{\mathrm{i}}{ }^{2}$ is therefore $\left(m_{\mathrm{i}} / m_{\mathrm{e}}\right)^{0.5}=43-76$ times larger than $\chi_{\mathrm{e}}^{\mathrm{nc}}$ and one can expect values up to $1 \mathrm{~m}^{2} / \mathrm{s}$.

$\mathrm{f}$ Parallel resistivity should be given by Spitzer's formula enhanced by the presence of trapped electrons with the factor $\left(1-(r / R)^{0.5}\right)^{-2}$. g A part of the toroidal current is driven by the radial density and temperature gradients: the so-called bootstrap current, in first order given by $j_{\mathrm{bc}}=-(r / R)^{0.5}\left(T / B_{\mathrm{pol}}\right) \nabla n$. Values around $10^{5} \mathrm{~A} / \mathrm{m}^{2}$ can be expected.

$\mathrm{h}$ Viscosity for flows in the poloidal direction should be very high, effectively limiting poloidal velocities to typical $10^{3} \mathrm{~m} / \mathrm{s}$.

i Viscosity for flows in the toroidal direction should be low with a momentum diffusivity $\chi_{\phi} \sim \chi_{i}^{\text {nc }}$.

\section{I.B. Transport in Case of Electrostatic Turbulence}

This subject will be treated extensively in this course [3]. The main idea is that fluctuations in the electrostatic potential due to instabilities cause particle drifts perpendicular to the further undisturbed magnetic surfaces. This enhances transport anomalously above the neoclassical level. Various instability models have been proposed each with their growth rate $\gamma$ and typical wave number $k_{\perp}$.

I.C. Transport in Case of Magnetic Turbulence

In case magnetic field lines cannot be described by closed magnetic surfaces one has to expect a stochastic meandering of field lines. Particles bound by these field lines have a finite chance that they reach the edge of the plasma. Since $\chi_{\|}$is many orders of magnitude higher than $\chi_{\perp}$ this is a very effective loss mechanism especially for electrons. The predicted electron heat diffusivity is given by:

$$
\chi_{\mathrm{e}}^{\mathrm{an}}(\perp)=(\tilde{B} / B)^{2} \chi_{\mathrm{e}}^{\mathrm{c}}(\|)
$$

From this we can conclude that a magnetic disturbance of $(\tilde{B} / B)=10^{-5}$ is already enough to get $\chi_{\mathrm{e}}>\chi_{\mathrm{e}}^{\mathrm{nc}}$. Particle diffusion is more complicated because a fast loss of electrons will only lead to strong electric fields withholding electrons and accelerating ions, i.e. lead to ambipolar flows.

\section{EXPERIMENTAL METHODOLOGY}

The experimental methodology to obtain insight in the possible transport mechanisms is to measure values belonging to the scheme of four categories shown below and compare the results of these categories: 


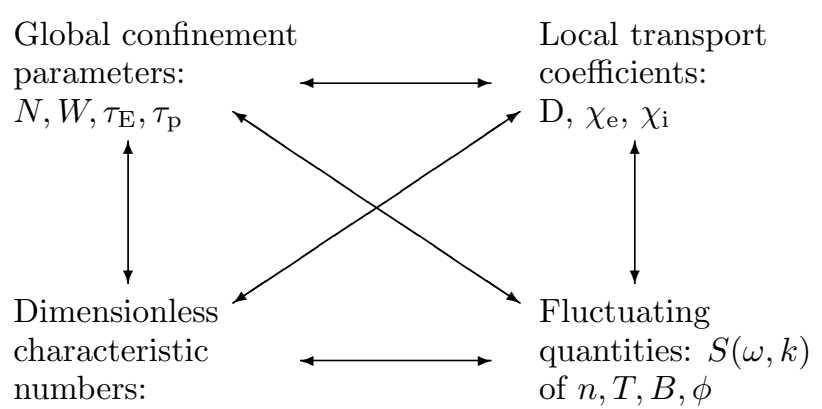

$\nu^{*}, \beta, \eta$, magn.Reynolds, etc.

Quantities like $\chi_{\mathrm{e}}$ are derived from the so-called balance equations. These describe the plasma state as the result of balances between sources, sinks and fluxes. They can be derived by integrating various moments of the kinetic equation over velocity distributions. For practical experimental purposes one can limit oneself to three of these moments, resulting in the particle balance or continuity equation, the momentum balance, and the energy balance. As an example, the particle balance can be written as:

$$
\partial n / \partial t+d i v \cdot \Gamma=\text { Sources }- \text { Sinks }
$$

where the particle flux consists of a diffusive and a convective part:

$$
\Gamma=-D \nabla n+n V_{\mathrm{c}}
$$

The balances are made up of numerous terms; usually a numerical transport code, with modules for the various source terms, is needed to solve the coupled balance equations - see the lecture on this subject [4].

\section{II.A. Global Confinement Quantities}

These are based on the plasma volume integral of density and energy, i.e. the total particle and energy content $(N, W)$. The particle and energy losses can be written as $N / \tau_{\mathrm{p}}$ and $W / \tau_{\mathrm{E}}$, respectively, in which $\tau_{\mathrm{p}}$ and $\tau_{\mathrm{E}}$ are the characteristic relaxation times for particle and energy confinement lumping all loss mechanisms together. The balance equations can be simplified to:

$$
\begin{array}{r}
\tau_{\mathrm{p}}{ }^{*}=\tau_{\mathrm{p}} /(1-R)=N /\left(\Sigma \Phi_{\mathrm{ext}}-\partial N / \partial t\right) \\
\tau_{\mathrm{E}}=W /\left(\Sigma P_{\mathrm{ext}}-\partial W / \partial t\right)
\end{array}
$$

where $\Phi_{\text {ext }}$ and $P_{\text {ext }}$ denote the external particle and heat sources, respectively, and where $R$ is the recycling coefficient.

\section{II.B. Local Transport Coefficients}

Local transport coefficients are derived from experiments by calculating the fluxes crossing every magnetic surface $\Psi$ and relating them to the local gradients at $\Psi$. From transport theory it follows that very probably every flux is driven by more than one gradient. Therefore. one has to solve a matrix equation of the type:

$$
\left(\begin{array}{l}
\Gamma \\
q
\end{array}\right)_{\Psi}=-\left(\begin{array}{cc}
D & M_{12} \\
M_{21} & n \chi
\end{array}\right)\left(\begin{array}{c}
\nabla n \\
\nabla T
\end{array}\right)_{\Psi}
$$

in this example simplified to a two-by-two matrix. The fluxes crossing the magnetic surface $\Psi$ can be calculated if one knows how large fraction of the external particle and heating sources are deposited within surface $\Psi$, i.e. $\Phi_{\text {ext }}(\Psi)$ and $P_{\text {ext }}(\Psi)$. This has to be corrected for the sinks integrated over the volume within $\Psi$ and the change in particle and energy content within $\Psi$ :

$$
\left(\begin{array}{l}
\Gamma \\
q
\end{array}\right)_{\Psi}=\frac{1}{\text { Area }}\left(\begin{array}{c}
\Sigma \Phi_{\text {ext }}(\Psi) \\
\Sigma P_{\text {ext }}(\Psi)
\end{array}\right)-(\operatorname{Sinks})-\left(\begin{array}{l}
\dot{N}(\Psi) \\
\dot{W}(\Psi)
\end{array}\right)
$$

Special approaches to this scheme (see also Fig.1):

a Neglect off-diagonal transport matrix elements $\left(M_{i j}=0\right.$ for $\left.i \neq j\right)$; the resulting diagonal transport coefficients are called $D^{\text {eff }}, \chi^{\text {eff }}$, etc.

b One limits oneself to semi-stationary situations: $\partial N / \partial t=0 ; \partial W / \partial t=0$.

c One deliberately modulates $N$ and $W$ to derive dynamic values of $D, \chi$ from the propagation velocity of particle and heat waves: $D^{\mathrm{dpp}}, \chi^{\mathrm{hpp}}$, etc. [5].

$\mathrm{d}$ In case one finds a nonlinear relation between flux and gradient one replaces $D^{\text {eff }}, \chi^{\text {eff }}$ by the incremental values: $D^{\text {inc }}=\partial f$ lux $/ \partial$ (gradient). This approach is sketched in Fig.1. Nearly all experimental results show $D^{\text {inc }}=D^{\text {dpp }} ; \chi_{\mathrm{i}}^{\text {nc }}=\chi^{\text {hpp }}$, etc.

Deriving local transport coefficients from experimental profiles is based on two assumptions. First, these coefficients should not vary strongly on the length scale of the observation methods used. Second, the transport coefficients should be constant on surfaces of equal $\Psi$. The existence of Internal Transport Barriers (narrow regions of reduced transport) may falsify the first assumption. Since many kinds of turbulence have ballooning nature, i.e. they are localized on the outboard side, also the second assumption is questionable.

\section{II.C. Dimensionless Parameters}

Each of the various transport models depends on a different set of characteristic dimensionless numbers. For instance neoclassical transport depends critically on the so-called collisionality, i.e. the ratio of one banana orbit length and the collision free mean path of the averaged particle, etc. The reader is referred to the various lectures on transport theory of this summer school $[1,2,3]$.

\section{II.D. Fluctuations}

Ideally one wants to know fluctuation levels of $n, T, B$ and electrostatic potential $\Phi$ as well as their wavelength, frequency, growth rate and phase correlation between them. An overview of measurement techniques for these parameters is given in [6]. 

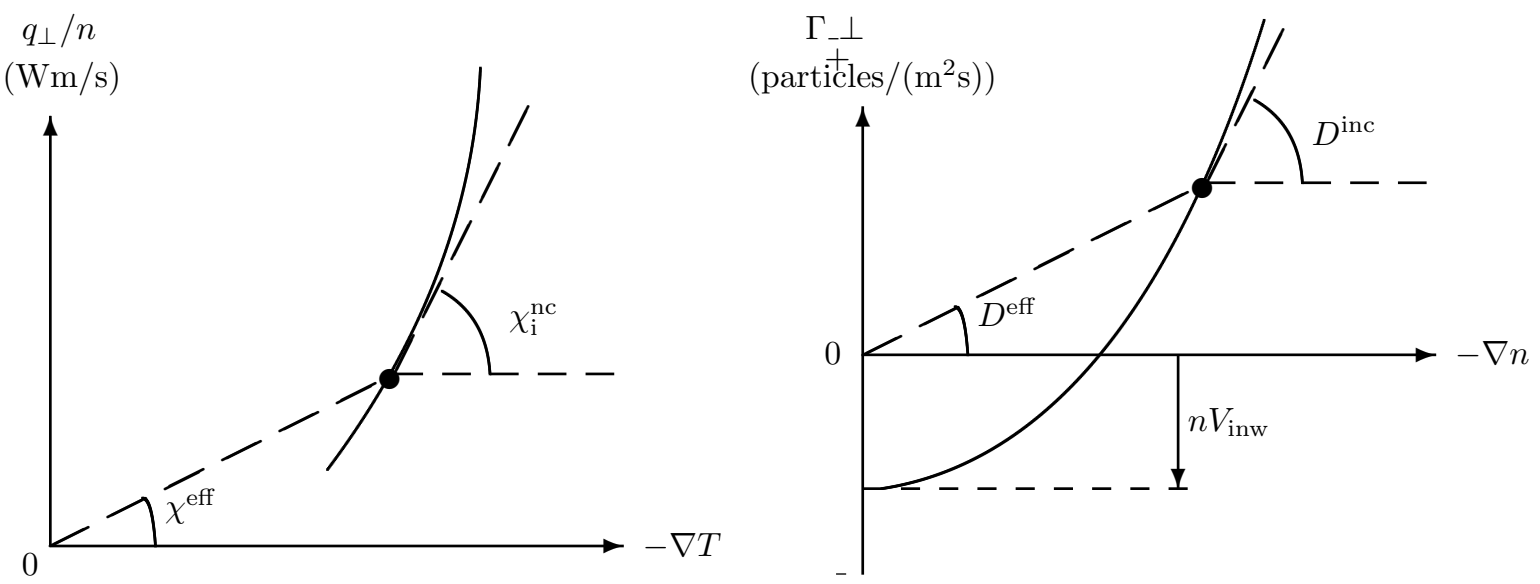

Figure 1: Schematic dependence of flux and gradient with the definitions of transport coefficients as given in the text. Anomalous inward particle and heat fluxes lead to an off-set crossing of the zero flux line and could be caused by other gradients than the prime one used for the horizontal axis. For instance in case of the particle flux a clear inward velocity has been observed presumably driven by the temperature gradient.

\section{EXPERIMENTAL RESULTS WITH OHMI- CALLY HEATED PLASMAS}

In tokamak experiments with only ohmic heating 3 types of confinement behaviour occur:

a At low densities the plasma temperature stays nearly constant with increasing density, i.e. $\tau_{\mathrm{E}} \sim$ $n_{\mathrm{e}}$. This is called $\mathbf{L O C}=$ 'Linear Ohmic Confinement'. Comparing experiments with different sizes, magnetic field and current the so-called NeoAlcator scaling was established:

$$
\tau_{\mathrm{E}}=7 \cdot 10^{-22}\langle n\rangle a R^{2} q_{a}^{0.5}
$$

This scaling is in stark contrast with neoclassical theory, which predicts $\tau_{\mathrm{E}}^{\mathrm{nc}}$ to fall with $n_{\mathrm{e}}$. At low density $\tau_{\mathrm{E}}^{\mathrm{nc}}$ is at least one order of magnitude higher than the experimental value. Local transport analysis showed that this is mainly caused by the anomaly in the electron heat transport: $\chi_{\mathrm{e}}$ is two orders of magnitude higher than $\chi_{\mathrm{e}}^{\mathrm{nc}}$ and this anomaly scales with $1 / n_{\mathrm{e}}$.

b The standard regime at high $n_{\mathrm{e}}$ is $\mathbf{S O C}=$ 'Saturated Ohmic Confinement', characterized by $\tau_{\mathrm{E}}$ being independent of $n_{\mathrm{e}}$. In this regime $\chi_{\mathrm{e}} \simeq \chi_{\mathrm{i}} \simeq$ $f \cdot \chi_{\mathrm{i}}^{\mathrm{nc}}$ with $f \simeq 1$ for small machines and $\simeq 3-10$ for large machines. The transition density between LOC and SOC appears to scale inversely proportional to the machine size.

c The $n_{\mathrm{e}}$ profiles in both LOC and SOC always show a modest peaking, only weakly dependent on $q_{a}$ with $n_{\mathrm{e}}(0) /\left\langle n_{\mathrm{e}}\right\rangle \simeq 1.5$. With a strong central particle source (by means of pellet injection) the peaking factor could be raised to more than 2 with the surprising result that LOC was restored at SOC densities. This regime is called $\mathbf{I O C}=$ 'Improved Ohmic Confinement', see Fig.2.

The observations indicate that there are two or even more independent mechanisms each causing anomalous transport with different scaling. One mechanism, responsible for LOC behaviour, acts on electrons only and is damped linearly with density. A second mechanism, only slightly influenced by density, acts on both ions and electrons alike. This was confirmed by density fluctuation levels which dropped dramatically with increasing density in LOC but leveled off to a low, but still significant constant value of a few percent in SOC. See also Section VI. The IOC transition suggests that the latter mechanism is related with the density gradient.

\section{EXPERIMENTAL RESULTS WITH ADDITION- ALLY HEATED PLASMAS}

The standard regime with strong additional heating is the so-called L-mode = 'Low confinement mode', where confinement is degraded even more beyond SOC. Main observations:

- an increase of $W$ with only $P_{\text {tot }}{ }^{0.5}$ and therefore $\tau_{\mathrm{E}} \sim$ $P_{\text {tot }}{ }^{-0.5}$;

- $\tau_{\mathrm{E}}$ is nearly independent of density like SOC;

- a near linear increase in $W$ and $\tau_{\mathrm{E}}$ with current;

- the same profile consistency as with LOC and SOC.

A schematic behaviour of $\tau_{\mathrm{E}}$ with density and power for ohmic and additionally heated plasmas is shown in Fig.2.

IV.A. Scaling Laws

Since L-mode behaviour is of crucial importance for the 


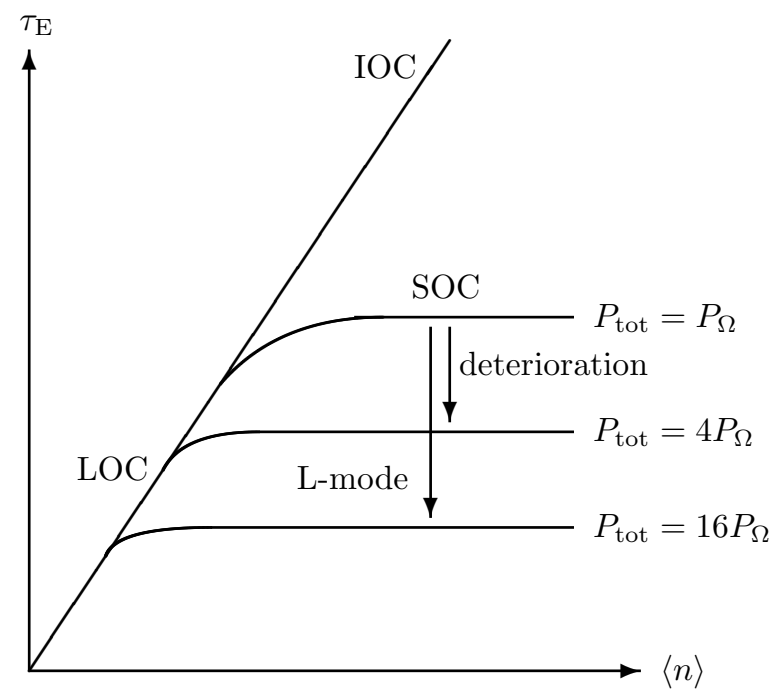

Figure 2: Scheme of the behaviour of $\tau_{\mathrm{E}}$ with density for LOC, $S O C, I O C$ and L-mode.

design of a thermonuclear reactor, the ITER confinement database working group constructed with great care a database of all existing tokamak results ranging of experiments with a typical size of $0.1 \mathrm{~m}$ to large ones with dimensions of meters and input powers ranging from 0.3 to $22 \mathrm{MW}$. Based on the subset of 1800 L-mode discharges (no ohmic discharges) the following scaling was found [7]:

$$
\tau_{\mathrm{E}}=0.037 I_{p}^{0.74} B^{0.2} \kappa^{0.67} R^{1.38} a^{0.31} n^{0.24} M^{0.26} P^{-0.57}
$$

This scaling predicts experimental results with an accuracy of $15 \%$ notwithstanding the huge span in conditions. This suggests that one mechanism is responsible for L-mode behaviour. It is comforting that also small tokamaks can study L-mode behaviour and still obtain reactor relevant results.

\section{IV.B. Local Transport}

The deterioration of confinement in L-mode plasmas is reflected in the local heat diffusivities. In general, $\chi_{\mathrm{e}}$, $\chi_{\mathrm{i}}$ and $\chi_{\phi}$ are close to each other. At high power $\chi_{\mathrm{i}}$ can be more than one order of magnitude larger than $\chi_{i}^{\mathrm{nc}}$.

Dynamic measurements via perturbative methods, i.e. simultaneous heat- and particle pulse propagation show that off-diagonal elements in the transport matrix are important. For instance, the particle flux is strongly influenced by temperature gradients. See lecture on perturbative transport [5].

Off-axis heating has been used (JET) to study the dependence between the total heat flux and the electron temperature gradient. The result is as sketched in Fig.1a, i.e. without an inward heat pinch. However, in DIII-D one has made a distinction between ion and electron heat flux for off-axis ECRH discharges. The authors claim that then a heat-pinch effect in the electron heat flux shows up which is not present in the total heat flux [8]. The dependence of $q_{e}$ vs. $\nabla T_{\mathrm{e}}$ is then similar to Fig.1b, rather than Fig.1a.

In TFTR one has compared pure deuterium and tritium plasmas. It has been found that $\chi_{\mathrm{i}}$ decreases more than inversely proportional with ion mass whilst $\chi_{\mathrm{e}}$ is independent of it. This explains why the global L-mode scaling of Eq.9, being a combination of both diffusivities shows an improvement of confinement with mass.

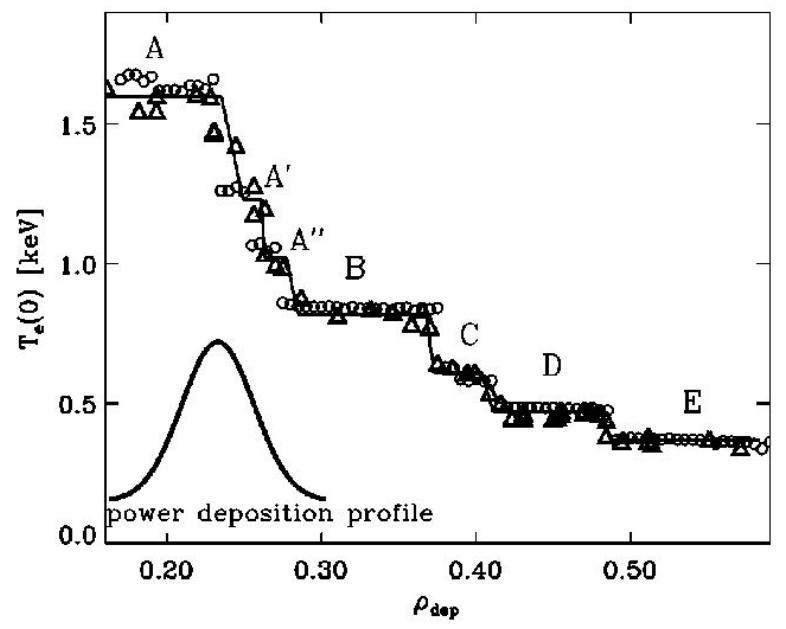

Figure 3: Central $T_{e}$ as function of deposition radius of ECRH $\left(\rho_{\mathrm{dep}}\right)$ for a series of similar discharges of RTP (taken from [9]). The jumps in $T_{e}(0)$ indicate the crossing of an internal transport barrier by $\rho_{\mathrm{dep}}$.

The sophisticated Thomson-scattering diagnostics at the RTP tokamak with a spatial resolution of $1 \%$ of the minor radius has resulted in the proof of existence of strong electron thermal barriers near radii with $q(r)$ close to an integer or half-integer value [9], see Fig.3. This suggests that L-mode confinement is in reality a global description of a very inhomogeneous and discontinuous plasma state with different ion- and electron transport mechanisms.

\section{CONDITIONS WITH IMPROVED CONFINE- MENT}

Several methods were found to improve confinement albeit often only during a limited dynamic phase. These improved confinement modes are not only the starting point for creating more permanent and favourable conditions, they also contain clues what the problem with L-mode confinement is.

V.A. H-mode and influence of shear in plasma rotation

Already in the early eighties ASDEX showed that improvements in confinement could be obtained in a divertor machine [10]. 
Necessary condition: the heat flux over the last closed magnetic surface should be above a minimum level. The threshold value for the heat flux across the last closed magnetic surface increases with density and magnetic field, but decreases with ion mass.

Improvement reached: in standard divertor $\mathrm{H}$ mode $\tau_{\mathrm{E}}^{H}=2 \tau_{\mathrm{E}}^{L}$. However, lately DIII-D has reached conditions for a VH-mode (Very High) which showed even an improvement of a factor 3.5. H-modes can be made semi-stationary by repetitive edge instabilities: ELMs. This causes a reduction in improvement to 1.75. This is the favoured mode for the plasma ignition device ITER. In limiter H-modes one normally reaches not more than a factor 1.5 .

Observations: the onset of the H-mode is characterized by the sudden development of a barrier for particle, momentum and heat flux at the last closed magnetic surface (LCMS). Therefore, a sudden jump in density and temperature gradient across the LCMS occurs, creating an edge pedestal in the profiles. Simultaneously, the neutral density in scrape-off layer and divertor plasma falls, leading to the very characteristic drop in $H_{\alpha}$ emission. Probe and Doppler-shift measurement show a dramatic change in the radial electric field and poloidal rotation. Many authors postulate that this change is the essential one. This is confirmed by the observation during the VH-mode that the change in the shear of the radial electric field is extended further inwards than in H-mode [11]. Also the fact that limiter H-modes could be obtained in TEXTOR by forcing a change in rotation with a radial current introduced by electrodes [12], points in this direction. In the edge barrier all transport coefficients are reduced simultaneously.

\section{V.B. Improvement by Peaked Density Profiles}

In Ohmic discharges IOC was reached by creating more peaked density profiles than in SOC. The same result can be obtained in additionally heated plasmas.

Necessary condition: a strong particle source on axis. This can be done in a continuous way by massive neutral beam injection by which TFTR has reached its 'supershot' regime or in a more dynamic situation by pellet injection as Pellet-Enhanced Performance ('PEP') mode in JET [13], see Fig.4.

Improvement: the higher the value of $n_{\mathrm{e}}(0) /\left\langle n_{\mathrm{e}}\right\rangle$, the better. Empirical result of supershots: $\tau_{\mathrm{E}}=$ $2 \tau_{\mathrm{E}}^{L}\left(n_{\mathrm{e}}(0) /\left\langle n_{\mathrm{e}}\right\rangle-1\right)$. Improvement of a factor 3 above L-mode has been reached with a density peaking of 2.5.

Observations: also here all transport coefficients are reduced in a similar way. This means that also the impurity transport is reduced and an accumulation of impurities can occur. The large $\nabla n_{\mathrm{e}}$ creates a large off-axis $j_{\mathrm{bc}}$, which may maintain an inverted $q$ profile and an inversion in magnetic shear. This magnetic con- figuration leads to improved confinement as will be discussed in subsection V.C. This raises the question if the high peakedness of the density in itself is the reason for the improved confinement or that this peakedness is only instrumental to changing the magnetic configuration by a large $j_{\mathrm{bc}}$.

\section{V.C. Improvement by Shear Inversion}

Normally the magnetic shear $(s)$ in tokamaks is positive, i.e. the angle between the total magnetic field and the toroidal direction increases faster with increasing minor radius $(r)$ than the ratio $r / R$ itself, i.e. $q$ increases from plasma centre to edge as a result of a monotonous decrease of the current density towards the edge.

Necessary conditions: In cases where the current density profile is hollow the value of $q$ first decreases going from axis to edge but increases again from a certain radius onwards (see Fig.4). The inner part has then negative shear (NCS, C for Central), sometimes also called reversed shear (RS). This configuration can be obtained transiently by making use of the skin current effect, i.e. ramping the plasma current up faster than the magnetic diffusion allows penetration. In steady state the same can be reached by strong counter-current drive on axis and/or co-drive off-axis. The bootstrap current helps since it is largest there where the density gradient is strongest, i.e. off-axis.

Observations: The effect of such configuration on confinement is stunning and since about 1995 confirmed by many machines, ranging from small (RTP) to large (JET, TFTR, JT-60, DIII-D) [14, 15, 16, 17]. Within the radius where $s<0$ all turbulence appears to be damped and confinement increases to neoclassical values. Record values of central plasma pressures have been reached. In some cases a local strong reduction of transport is observed only near the position of minimum $q$; in other cases a more global transport reduction inside $q_{\min }$ is seen; the term internal transport barrier (ITB) is used in both cases. It should be noted that there appears to be a difference between electron and ion thermal transport: whereas for the electrons RS is sufficient to create an ITB [18], for the ions a combination of RS and high input power, i.e. strong flow shear, is needed.

\section{V.D. Improvement by Impurity Injection}

In TEXTOR [19] and later on also in large tokamaks like DIII-D and JET [20, 21], it was shown that impurity (neon, argon) injection could enhance the confinement: the so-called RI-mode. Appreciable enhancements of more than a factor 2 have been quoted. The transition from L-mode to RI-mode is believed to be caused by suppression of long wave length turbulence, in particular the Ion Temperature Gradient 

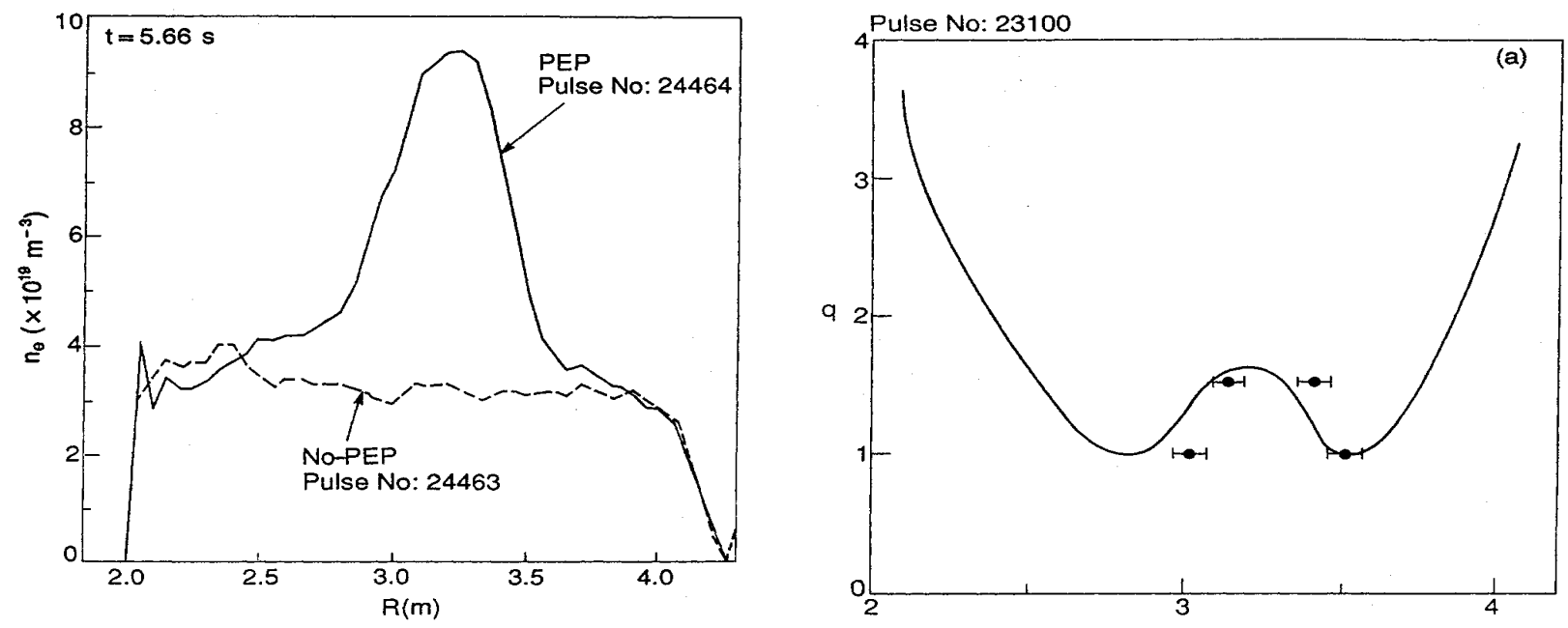

Figure 4: PEP mode in JET. Left panel: $n_{e}$ profile for a PEP discharge, compared with a no-PEP one. Right panel: $q$ profile, showing a region of reversed magnetic shear.

(ITG) mode [22]. The maximum growth rate of the ITG mode $\left(\gamma_{\mathrm{ITG}}^{\max }\right)$ decreases with $Z_{\text {eff }}$ and and the peaking of the $n_{\mathrm{e}}$ profile. The suppression mechanism is supposed to be as follows: $Z_{\text {eff }}$ is increased by impurity puffing; this decreases $\gamma_{\mathrm{ITG}}^{\max }$, which leads to peaking of $n_{\mathrm{e}}$, which in turn further decreases $\gamma_{\mathrm{ITG}}^{\max }$. In the end this leads to full suppression of the ITG mode.

Apart from the improvement of confinement, the RI-mode is attractive because the radiative mantle evenly distributes the heat load over the vessel, in stead of concentrating it on the limiter or in the divertor.

\section{V.E. Outlook for ITER}

Scenarios for ITER are developed along 2 lines: on one hand ELMy H-mode, on the other hand advanced scenarios based on NCS [23]. The latter have been vigorously pursued on JET; steady-state NCS operation appears possible with zero loop voltage, i.e. $j_{\mathrm{bc}}$ plus the current driven by the external heating sources are sufficient to sustain the desired plasma current [24]. Moreover, great progress has been made in JET towards real-time control of the plasma evolution [25].

\section{FLUCTUATIONS}

Fluctuation measurements confirm that tokamak plasmas are a turbulent fluid. Measurements in TEXT and Tore Supra (Fig.5) show that there are at least two mechanisms acting at the same time in SOC and Lmode plasmas:

1. A broad band turbulence with large potential and density fluctuations exists at the edge. Levels are several tens of \%. Electrostatic turbulence seems likely especially because the flux derived from $n$ and $E$ fluctuations are in good agreement with the local particle balance.
2. In the centre another broad band turbulence exists with high magnetic field fluctuations.

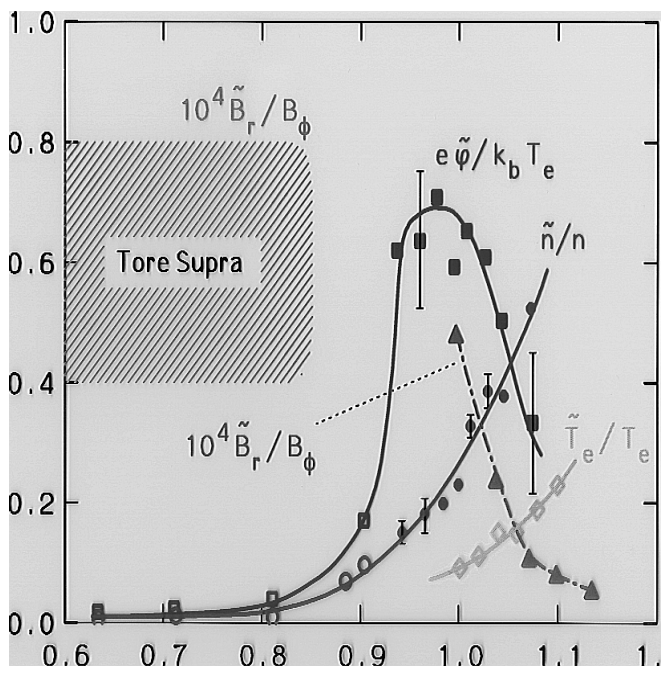

Figure 5: TEXT and Tore Supra results: relative level of various fluctuating quantities plotted against $r / a$.

The central density fluctuations are according to many experiments only on a few percent level but probably still significant, because the level is directly correlated with the anomaly in transport. The frequency spectrum is very similar to the magnetic fluctuations. High level temperature fluctuations can only be observed if their spatial dimension is equal or larger than the spatial resolution of the ECE equipment.

Interestingly, $\langle k \perp\rangle$ decreases with increasing size of the device in contradiction with the electrostatic turbulence theory predicting $\langle k \perp\rangle \cdot \rho_{\mathrm{i}} \simeq 1$. Values below 0.1 have been reported. This suggests that $\tau_{\mathrm{E}}$ should scale 

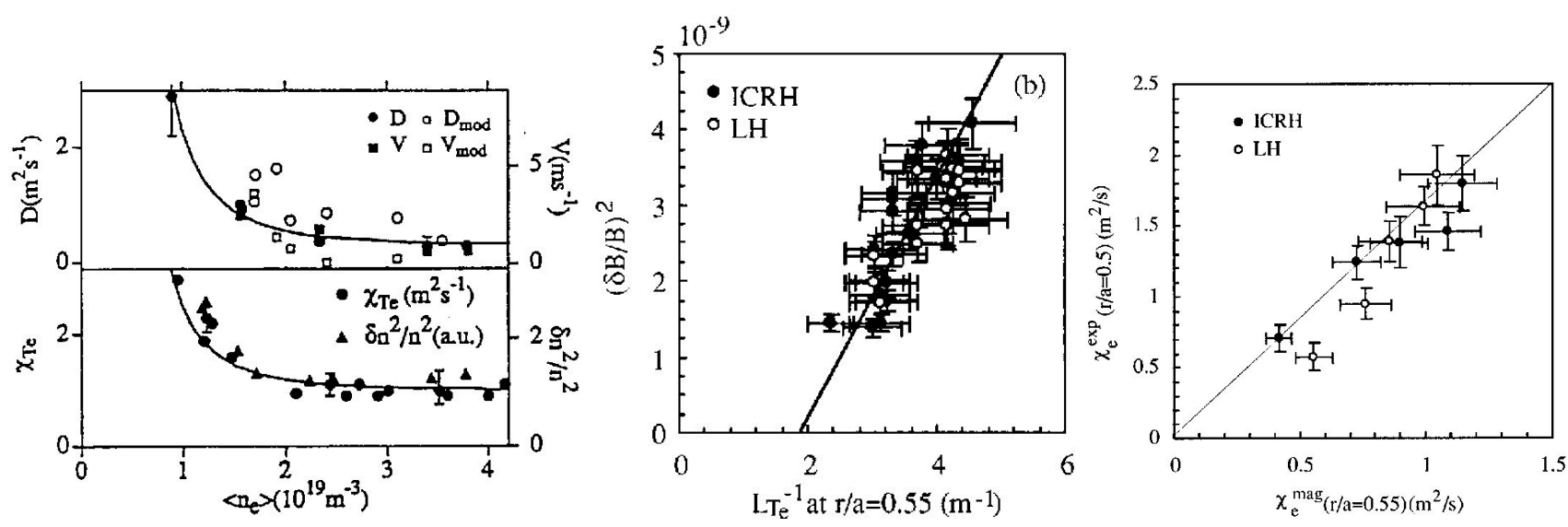

Figure 6: Relation between transport coefficients and fluctuation measurements in TORE SUPRA. Left: D and $\chi_{\mathrm{e}}$ as function of $n_{\mathrm{e}}$ in ohmic plasmas together with the fluctuation level $(\partial n / n)^{2}$. Middle/right: Magnetic fluctuation levels as function of $\beta_{\mathrm{pol}}$ (middle), and $\chi_{\mathrm{e}}$ calculated from this (right), taken from [26].

with $a / \rho_{\text {i }}$ on a 'Bohm'-like way, whilst electrostatic turbulence theory predicts so-called 'gyro-Bohm' scaling.

It is still unclear how potential fluctuations in the core fit into the observations.

In Section III it was already mentioned that in LOC plasmas probably a third mechanism plays a dominant role since there density fluctuations are on a much higher level than in SOC plasmas. This high level is reduced with increasing density in the linear regime until it reaches $\mathrm{SOC}$ level at the transition to the SOC regime (see Fig.6).

\section{VI.A. Connection with Transport}

Ideally, one would like to see quantitative agreement between the measured fluctuation level and the observed heat or particle transport. One example of this is the measurement of magnetic turbulence in Tore Supra, which indicated fluctuation levels of typically $5 \cdot 10^{-5}$, inducing the right level of electron heat transport [26], see Fig.6. This is a strong argument in favour of magnetic turbulence as driving force of anomalous electron heat transport.

Another important quantitative observation of TFTR and TORE SUPRA is that if one derives the growth rate from the frequency spectra of the density fluctuations and the average perpendicular wavelength from the $k$-spectra and one plugs that in the expression for strong turbulence diffusivities $\left(D=\chi=\gamma /\langle k \perp\rangle^{2}\right)$ one finds very good agreement with local diffusivities for all kinds of confinement regimes.

Qualitative agreement is another way to prove the role of fluctuations. This can be either in time, i.e. a sudden drop of the turbulence level at the transition to an enhanced confinement regime, or in radial position, i.e. turbulence level and transport level have related radial dependences. Either of this is a strong indicator that indeed the fluctuations were driving the (locally

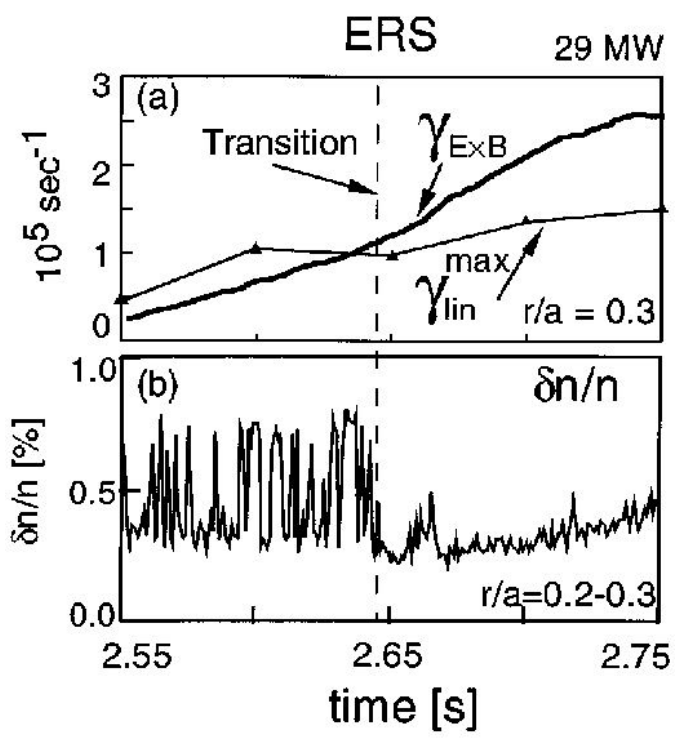

Figure 7: Transition from Reversed Shear to Enhanced Reversed Shear regime in TFTR (marked with dashed line), taken from [32]. Note the marked reduction of density fluctuation level at the transition.

or in time) quenched anomalous transport. There are many observations of this, e.g:

a The transition from $\mathrm{RS}$ to ERS in TFTR (E stands for Enhanced), see Fig.7.

b The observation that edge fluctuations in TEXTOR sharply drop at the transition to RI-mode [27].

c The electron ITB in electron heated JET discharges, where the fluctuation level sharply increases outside the ITB [28], see Fig.8.

\section{VI.B. Theoretical Modelling}

Modelling of turbulent transport can be done at different levels of detail, e.g. gyro-fluid vs. kinetic etc. 

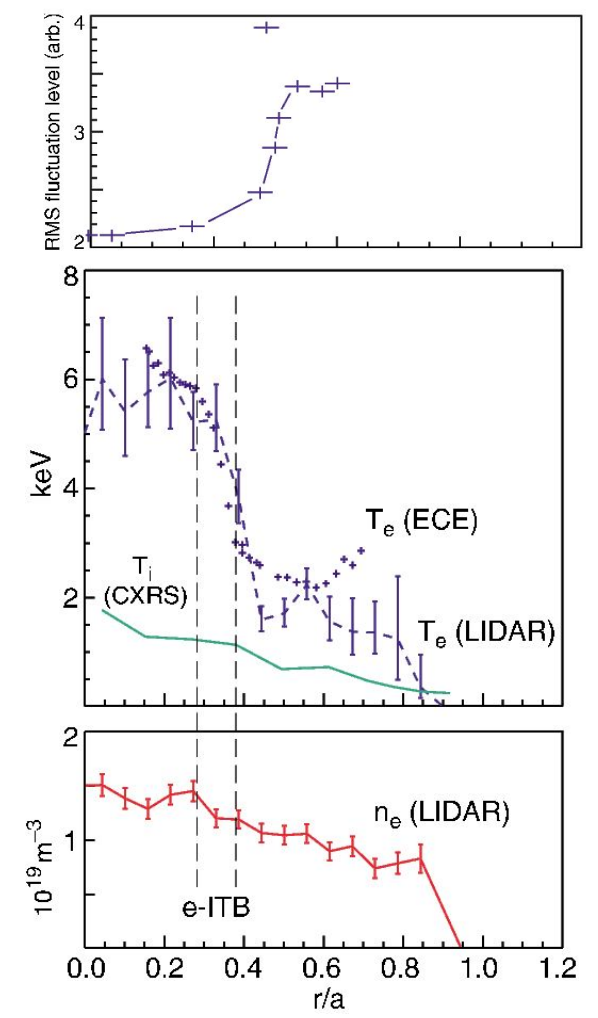

Figure 8: JET discharge with electron heating only. Upper panel: density turbulence level as measured with reflectometry; lower panel: $T_{e}$ profile (taken from [28]).

See for example $[29,30]$. Generally, turbulence is seen as $2 \mathrm{D}$ structures. This puts a strong request on diagnosticians to provide 2D measurements of fluctuations. Recent developments on TEXTOR promise to provide such $2 \mathrm{D}$ measurements of $\tilde{n}$ and $\tilde{T}$ by means of Microwave Imaging Reflectometry and ECE-Imaging.

Much of the turbulent transport modeling work does not take into account the magnetic structure of the plasma, although it is clear from the experimental results that the $q$ and $s$ profile are important. Some recent theoretical work, however, does reproduce the effect of rational $q$ and shear reversal [31].

\section{CONCLUSIONS}

1 Transport in tokamaks is of turbulent nature.

2 There are probably two or three mechanisms acting at the same time:

- in the edge there is a broad band turbulence where strong electrostatic fluctuations play a dominant role;

- in the centre there is at least one mechanism responsible for SOC- and L-mode behaviour with an important level of magnetic fluctuations and the $q$-related electron thermal barriers;

- in LOC discharges a third mechanism may play a role which nature is unknown but with rather strong central density fluctuations which are quenched at high density.

3 Turbulence causes particle, momentum and heat transport to vary simultaneously in a similar way.

4 There are strong non-diagonal elements in the transport matrix.

5 The effective diffusivities show a strong turbulence dependence: $D=\chi=\left(\gamma /\langle k \perp\rangle^{2}\right)_{\max }$

6 The turbulence level can be reduced under the following conditions:

- steepening of the $n_{\mathrm{e}}$ (IOC, supershot, RI-mode); - change of the shear in radial electric field/poloidal rotation (H- and VH-mode);

- magnetic shear inversion (NCS, PEP-mode).

7 With magnetic field fluctuations of $10^{-5}$ to $10^{-4}$, it appears doubtful if the concept of unbroken magnetic surfaces is still useful for transport theory and if the distinction between electrostatic and magnetic turbulence can be held up. Probably a unified theory is necessary in which the magnetic field topology is broken in the presence of potential fluctuations such that fluctuations of $B$ and potential mutually reinforce each other.

ACKNOWLEDGMENTS This work, supported by the European Communities under the contract of Association between EURATOM/FOM, was carried out within the framework of the European Fusion Programme with financial support from NWO. The views and opinions expressed herein do not necessarily reflect those of the European Commission.

\section{REFERENCES}

1. B. WEYSSOW, these Proceedings

2. P. HELANDER, these Proceedings

3. G. DIF-PRALADIER, these Proceedings

4. J. ONGENA, these Proceedings

5. G.M.D. HOGEWEIJ, these Proceedings

6. A.J.H. DONNÉ et al, Fus.Sc.Techn. 49 (2T) (2006), 367

7. S.M. KAYE et al, Nucl.Fus. 37 (1997), 1303

8. C.C PETTY et al, Nucl.Fus. 34 (1994), 121

9. M.R de BAAR et al, Phys.Plasmas 6 (1999), 4645

10. F. WAGNER et al, Phys.Rev.Let. 49 (1982), 1408

11. K.H. BURRELL et al, Phys.Plasmas 1 (1994), 1536

12. S. JACHMICH, Pl.Phys.Contr.Fus. 40 (1998), 1105

13. P. SMEULDERS et al, Nucl.Fus. 35 (1995), 225

14. F.M. LEVINTON et al, Phys.Rev.Let. 75 (1995), 4417

15. E.J. STRAIT et al, Phys.Rev.Let. 75 (1995), 4421

16. S. ISHIDA et al, Phys.Rev.Let. $\mathbf{7 9}$ (1997), 3917

17. F.X. SÖLDNER et al, Pl.Phys.Contr.Fus. 39 (1997), B353

18. G.M.D. HOGEWEIJ et al, Pl.Phys.Contr.Fus. 44 (2002) 1155

19. R.R. WEYNANTS et al, Nucl.Fus. 39 (1999), 1637

20. G.L. JACKSON, Pl.Phys.Contr.Fus. 44 (2002), 1893

21. P. DUMORTIER, Pl.Phys.Contr.Fus. 44 (2002), 1845

22. M.Z. TOKAR et al, Phys. Rev.Let. 84 (2000), 895

23. E.J. DOYLE et al, Nucl.Fus. 47 (2007), S18

24. X. LITAUDON, Pl.Phys.Contr.Fus. 44'(2002), 1057

25. D. MOREAU et al, Nucl.Fus. 48 (2008), 106001

26. L. COLAS et al, Nucl.Fus. 38 (1998), 903

27. L. BOEDO et al, Nucl.Fus. 40 (2000), 209

28. G. CONWAY, Pl.Phys.Contr.Fus. 44'(2002), 1167

29. C. ANGIONI et al Nucl.Fus. 49 (2009), 055013

30. C. HOLLAND et al, Phys.Plasmas 18 (2011), 056113

31. X. GARBET et al Phys.Plasmas 8 (2001), 2793

32. E.J. SYNAKOWSKI et al Phys.Plasmas 4'(1997), 1736 